\title{
Active Attenuation of Sound Radiation from a Circular Fluid-Loaded Plate
}

\author{
Lucyna Leniowska ${ }^{\dagger}$ and \\ Institute of Technology, Pedagogical University of RzeszCw, ul. Rejtana 16, 35-310 RzeszCw, Poland
}

\begin{abstract}
Ryszard Leniowski
Department of Automatic Control and Computer Science, Technical University of RzeszCW, W. Pola 2, 35-310 RzeszCW, Poland
\end{abstract}

(Received 24 January 2000; accepted 22 September 2000)

\begin{abstract}
This paper is concerned with the problem of suppressing the far-field sound pressure radiating from a circular plate located in a finite baffle and which interacts with a fluid. The sound field is reduced by applying a control force directly at a point on the plate surface. For the system under consideration a state - space model is constructed. The optimal control problem is solved by including an additional term in the performance which is proportional to the squared farfield sound pressure radiated, in addition to the customary two terms which depend on the vector of state variables and on the control effort. The control input that minimises this performance is derived by applying Hamilton's principle.
\end{abstract}

${ }^{\dagger}$ Member of the International Institute of Acoustics and Vibration (IIAV)

\section{INTRODUCTION}

The problem of suppressing the far-field sound pressure radiated from planar sources such as plates is often solved by the application of active methods. A large number of studies on active noise control have been reported in recent years. In these studies, classical control, feed-forward control, modern control and robust control have been used. ${ }^{1}$ However, there are only a few papers where the interaction of sound waves with an elastic vibrating plate surface is taken into account. ${ }^{2,3}$ On the other hand, it is well known that for structures radiating into a relatively dense fluid the effect of the radiated sound field on the structural response cannot be ignored. As a result of the fluid coupling the response of the plate in the fluid can be significantly different from its responses in a vacuum or in air.

Another problem arises from the fact that vibrating plates are usually characterised by low-frequency harmonic vibrations. This means that it can be very difficult to satisfy the ideal condition of an 'infinite baffle', which is often used in calculations of acoustical quantities, because of constructional reasons. To develop successfully an effective solution for the problem of suppressing the far-field sound pressure, it is necessary to take into account the phenomena described above in the mathematical model of the system considered. Thus, in this paper it is assumed that the plate in question is located in a finite baffle and that it interacts with a relatively 'dense' fluid.

One common approach for the attenuation of the sound radiation from a plate is to suppress the plate vibration (vibration control). However, such attempts to control only a few plate modes individually may not lead to a reduction in the sound radiation because various modes have differing radiation efficiencies.
An alternative approach is to apply control inputs directly to the structure with the objective of reducing the overall sound radiation. In this case a cost function is derived from the far-field radiated sound pressure (radiation control). Thus, for the closed-loop system response, the overall radiation efficiency is reduced while the overall plate vibration amplitude is not satisfactorily attenuated or sometimes is increased. Hence, it is essential to select the modes to be controlled so as to minimise both the far-field sound pressure and the plate vibration simultaneously. This result is possible through the application of linear-quadratic control theory, if the minimised performance index (cost function) is chosen in an appropriate way.

The system chosen for investigation is a thin, circular plate clamped in a finite rigid coplanar baffle. The plate which is excited on one side by a uniform periodic force with a constant amplitude, radiates into free space. A force input located at a point at the middle of the plate seems to be quite an effective kind of control for such a system. ${ }^{4}$ The method for obtaining the optimal control force is based on the method proposed by Meirovitch. ${ }^{3}$ In order to design an optimal controller, the equation of motion of a fluid-loaded plate driven by a primary external force is expressed in the statespace form. The determination of the sound pressure is based on the admissible functions for a homogeneous plate in $v a$ cuo and on the properties of oblate spheroidal co-ordinates. Modern control theory is applied to reduce the response of the system in the way desired. The control law is chosen to minimise the performance index, which contains three components that are dependent quadratically on the vector of state variables, the control effort and on the far-field sound pressure radiated. The optimal control input that minimises this performance index is derived by applying Hamilton's principle. Finally, the simulation results obtained for an aluminium plate are presented. 